\title{
Treatment of digestive angiectasia: time for prospective, randomized, therapeutic studies
}

\section{다 (1) $\odot$}

\author{
Authors \\ Jean-Christophe Saurin, Philippe Jacob, Mathieu Pioche \\ Institution \\ Gastroenterology Unit, E. Herriot Hospital, Hospices Civils \\ de Lyon, Lyon, France \\ Bibliography \\ DOI https://doi.org/10.1055/a-0990-9773 | \\ Endoscopy International Open 2019; 07: E1778-E1779 \\ (c) Georg Thieme Verlag KG Stuttgart · New York \\ eISSN 2196-9736
}

\author{
Corresponding author \\ Jean-Christophe Saurin, Gastroenterology Unit, E. Herriot \\ Hospital, Hospices Civils de Lyon, 5 Place d'Arsonval, 69437 \\ Lyon cedex 03, France \\ Fax: +33472110147 \\ Jean-Christophe.saurin@chu-lyon.fr
}

Digestive angiodysplasia (or angiectasia, or arteriovenous malformations) is a frequent digestive finding. Three to $6 \%$ of colonoscopies are performed for reasons other than bleeding, $25 \%$ when systematic analysis of right hemicolectomy is performed $[1,2]$ On the other hand, colonic angiectasia almost never bleeds when discovered by chance, and is an uncommon cause of lower digestive bleeding [3].

Interestingly the natural history of bleeding from colonic angiectasia is that of a spontaneous bleeding cessation in more than half of patients during follow-up [4]. The same situation exists for small bowel angiodysplasia, so that the effectiveness of any therapeutic drug or endoscopic treatment is relatively difficult to demonstrate. For example, after a first very positive crossover study, estrogen proved to be clearly ineffective [5] In contrast to drug therapy, the clinical efficacy of endoscopic therapy for angiodysplasia has never been evaluated in a randomized placebo-controlled study, but only through (prospective or retrospective) cohorts, thus leaving significant room for doubt about its efficacy, given the natural history of bleeding angiectasia. This is the case not only with colonic angiectasia, which had an $89 \%$ bleeding cessation rate 18 months after argon plasma coagulation (APC) in one large retrospective series, [6] but also with small bowel angiectasia after endoscopic (usually also APC) treatment. In retrospective series, rebleeding rates of $42 \%$ to $56 \%$ have been seen at 55 months and 2 years, respectively $[7,8]$. Therefore, the evidence base is far from demonstrating any clear benefit for endoscopic treatment of bleeding digestive angiectasia. Still, most gastroenterologists are convinced that endoscopic treatment is mandatory in patients with anemia or overt bleeding from digestive angiectasia.

Given the lack of prospective demonstration of any benefit of endoscopic treatment, are there physiological data to clarify the efficacy of these treatment approaches? Nd-Yag Laser has long been the most-evaluated treatment for vascular digestive lesions but is now has been abandonned. Argon plasma coagulation (APC) replaced Nd-Yag in the early 2000 s, and few physiological studies are available. One evaluation on pig colonic wall showed a risk of transmural coagulation when prolonged (3 sec) APC application was used [9]. No histological results with APC for digestive angiectasia have been published, but risk of complication is estimated at $0.5 \%$ to $2.8 \%$ [6]. Japanese teams have recently proposed use of polidocanol to treat digestive hemangiomas, with impressive results and no complications in specific series of patients $[10,11]$. Japanese physiological studies have shown that polidocanol injection has two successive effects: the first early, pressure-related, and resulting in thrombotic effects on small vessels, and the second late thrombus formation effect secondary to vessel inflammation. Histology showed that the effects of polidocanol were limited to the submucosal layer [12]. Polidocanol injection has been used for a long time in a large number of patients and proven to be safe. But a few old case reports, in a very specific situation (deep duodenal ulcer and treatment with large volume polidocanol) reported some risk of polidocanol that may be related to digestive perforation [13]. On the other hand, extended experience proved this treatment to be safe in several randomized trials for prevention of ulcer bleeding [14].

As stated by the authors of this paper, digestive angiodysplasia is anatomically composed of a dilated submucosal vein associated with a mucosal arteriovenous malformation. Thus, superficial treatment of the mucosal part of angiodysplasia may explain the lack of clear efficacy of usual endoscopic treatments like APC $[15,16]$.

The new therapeutic protocol described by Tai et al clearly responds to the physiology of digestive angiodysplasia, by facil- 
itating efficient treatment of the responsible submucosal vessels. This approach is very innovative, appears feasible from the six reported cases, and seems efficient as far as can be ascertained from a six-case series with short follow-up. Will this treatment prove more efficient than previous ones? Possibly, but prospective series are needed and, as discussed above, at least some with randomization to alternative treatments. Might this treatment be safer than previous ones like APC or polidocanol? That absolutely needs to be demonstrated in prospective randomized studies. Indeed, there is a large published experience with forceps-coagulation, mainly in treatment of diminutive polyps, and the rate of complication is well established at $0.5 \%$ (bleeding and perforation) [17]. For these reasons, this approach for diminutive polyps is usually contraindicated nowadays. Use of this hemostatic technique in a large series of patients with colonic (or extra-colonic) angiectasia will certainly show some significant risks.

Bleeding from digestive angiectasia is a frequent clinical problem, especially for teams dealing with capsule endoscopy and enteroscopy. The efficiency of different available endoscopic treatments for it, however, has been very poorly evaluated. It is really time to promote some large, prospective, randomized studies comparing these treatments with simple medical management to clarify the optimal risk:benefit approach for patients with angiodysplasia.

\section{Competing interests}

The authors have received consultant fees from Medtronic and Intromedic.

\section{References}

[1] Hochter W, Weingart J, Kuhner W et al. Angiodysplasia in the colon and rectum. Endoscopic morphology, localisation and frequency. Endoscopy 1985; 17: $182-185$

[2] Heer M, Sulser H, Hany A. Angiodysplasia of the colon: an expression of occlusive vascular disease. Hepatogastroenterology 1987; 34 : $127-131$
[3] Boley SJ, DiBiase A, Brandt LJ et al. Lower intestinal bleeding in the elderly. Am J Surg 1979; 137: 57-64

[4] Richter JM, Christensen MR, Colditz GA et al. Angiodysplasia. Natural history and efficacy of therapeutic interventions. Dig Dis Sci 1989; 34: $1542-1546$

[5] van Cutsem E, Rutgeerts P, Vantrappen G. Treatment of bleeding gastrointestinal vascular malformations with oestrogen-progesterone. Lancet 1990; 335: $953-955$

[6] Olmos JA, Marcolongo M, Pogorelsky V et al. Argon plasma coagulation for prevention of recurrent bleeding from $\mathrm{Gl}$ angiodysplasias. Gastrointest Endosc 2004; 60: 881-886

[7] May A, Friesing-Sosnik T, Manner $\mathrm{H}$ et al. Long-term outcome after argon plasma coagulation of small-bowel lesions using double-balloon enteroscopy in patients with mid-gastrointestinal bleeding. Endoscopy 2011; 43: 759- 765

[8] Landi B, Cellier C, Gaudric M et al. Long-term outcome of patients with gastrointestinal bleeding of obscure origin explored by push enteroscopy. Endoscopy 2002; 34: $355-359$

[9] Norton ID, Wang L, Levine SA et al. In vivo characterization of colonic thermal injury caused by argon plasma coagulation. Gastrointest Endosc 2002; 55: 631-636

[10] Igawa A, Oka S, Tanaka S et al. Polidocanol injection therapy for smallbowel hemangioma by using double-balloon endoscopy. Gastrointest Endosc 2016; 84: $163-167$

[11] Igawa A, Oka S, Tanaka S et al. Major predictors and management of small-bowel angioectasia. BMC Gastroenterol 2015; 15: 108

[12] Takeuchi T, Narimiya N, Joki M et al. Experimental study on the effects of local injection of aethoxysklerol for gastric mucosa with special reference to the submucosal arterial plexus. Gastroenterol Endosc 1991; 33: $695-704$

[13] Opacic M, Pulanic R, Vucelic B et al. Massive necrosis of the gastric wall with gastric perforation after injection therapy of a bleeding duodenal ulcer. Endoscopy 1995; 27: $707-708$

[14] Llach J, Bordas JM, Salmeron JM et al. A prospective randomized trial of heater probe thermocoagulation versus injection therapy in peptic ulcer hemorrhage. Gastrointest Endosc 1996; 43: 117-120

[15] Sami SS, Al-Araji SA, Ragunath K. Review article: gastrointestinal angiodysplasia - pathogenesis, diagnosis and management. Aliment Pharmacol Ther 2014; 39: 15-34

[16] Boley S], Sammartano R, Brandt L] et al. Vascular ectasias of the colon. Surg Gynecol Obstet 1979; 149: 353 -359

[17] Weston AP, Campbell DR. Diminutive colonic polyps: histopathology, spatial distribution, concomitant significant lesions, and treatment complications. Am J Gastroenterol 1995; 90: 24-28 